\title{
Dumbbell fluidic tweezers for dynamical trapping and selective transport of microobjects
}

Qi Zhou, Tristan Petit, Hongsoo Choi, Bradley J. Nelson, and Li Zhang*

Q. Zhou, Prof. L. Zhang

Department of Mechanical and Automation Engineering

The Chinese University of Hong Kong

Shatin, N.T., Hong Kong, China

E-mail: lizhang@mae.cuhk.edu.hk

T. Petit, Prof. B. J. Nelson

Institute of Robotics and Intelligence Systems (IRIS)

ETH Zurich

CH-8092 Zurich, Switzerland

Prof. H. Choi

Robotics Engineering Department

Daegu Gyeongbuk Institute of Science and Technology (DGIST)

711-873, Daegu, South Korea

Prof. L. Zhang

Shenzhen Research Institute

The Chinese University of Hong Kong

Shenzhen, China

\section{Present address}

Q. Zhou

Institute for Materials and Processes

School of Engineering, University of Edinburgh

Edinburgh EH9 3FB, Scotland, United Kingdom

T. Petit

Helmholtz-Zentrum Berlin für Materialen und Energie

Albert-Einstein-Strasse 15, 12489 Berlin, Germany 


\begin{abstract}
Mobile microvortices generated by rotating nickel (Ni) nanowires have been reported able to induce fluidic trapping that can be precisely focused and translated to manipulate microobjects. Here, we report a new design for significantly enhanced fluidic trapping, which is a dumbbell-shaped magnetic actuator, assembled by a Ni NW and two polystyrene (PS) microbeads. In contrast to the single mode of tumbling trapping possessed by Ni NW, the magnetic dumbbell (DB) is able to perform dynamical trapping and implement on-demand transport of microobjects in three modes, i.e., tumbling, wobbling, and rolling. Experiments are conducted to demonstrate the robustness and efficacy of the fluidic trap by the DB actuator. And simulations using a finite element model compare the fluidic traps induced by NW and DB, followed by further discussion on the actuation and transport efficiency of NW and DB fluidic tweezers (FT). At last, some practical issues regarding the application of DB FT are addressed.
\end{abstract}

Keywords: dumbbells, fluidic trapping, microvortices, low Reynolds number flows, magnetic actuation, noncontact manipulation 


\section{Introduction}

Microrobots activated by magnetic fields have drawn extensive attention in recent years due to their promising potential for micromanipulation and biomedical applications. ${ }^{[1-6]}$ Frequently, these tasks require not only precise and controlled locomotion of the microrobot, but also its capability of cargo transport in biofluidic environments. To this end, two common strategies have been exploited: directly pushing objects with or loading them on the microrobot itself, ${ }^{[7-13]}$ and trapping objects in microflows/microvortices induced by the motion of the microrobot. ${ }^{[14-19]}$ The former strategy, generally referred to as contact manipulation, has been extensively reported in the literature. This paper aims to address the latter: non-contact manipulation.

A key aspect of manipulating micro-/nanoscale objects is to trap them. Traditional techniques such as optical tweezers (OT), ${ }^{[20-22]}$ magnetic tweezers $(\mathrm{MT}),{ }^{[23-25]}$ and dielectrophoresis (DEP), ${ }^{[26,27]}$ have long been used to accomplish this task. However, for biological samples, the concern is that the high intensity lasers, direct physical attachment (i.e., bonding to a magnetic particle) or strong electric fields in the above trapping techniques can have an impact on the viability of the samples. To evade these negative effects, alternative non-contact manipulating techniques were developed, relying on near field photonics, ${ }^{[28-30]}$ electrostatics, ${ }^{[31]}$ electro-kinetics, ${ }^{[32,33]}$ or acoustic waves. ${ }^{[34]}$ They have their own limitations though, the major one being providing only static trapping on fixed positions (one exception was recently made by Ndukaife et al. ${ }^{[30]}$ who achieved a long-range vortex using hybrid electrothermoplasmonic nanotweezer to transport objects to specific plasmonic nanoantennas). To our interest, another non-contact approach, hydrodynamic tweezers (HT), achieves particle trapping through frequency-dependent steady streaming induced by fluid oscillation near microfabricated geometries such as cylindrical obstructions, ${ }^{[35-37]}$ and wall protrusions or cavities. ${ }^{[38]}$ In comparison with the conventional OT, MT and DEP techniques, HT and some other similar techniques provide a non-invasive means to manipulate cells or 
microorganisms in low Reynolds number flow utilizing gentle but robust microeddies. ${ }^{[39-41]}$ Nevertheless, due to the geometric restrictions for HT to trap particles, only static immobilization is possible, and selective transport of individual microobjects is still unlikely to be realized by HT.

More recently, Petit et al. found that microvortices similar to the streaming cells in HT were generated by the tumbling rotation of magnetic microactuators such as a Ni NW or a self-assembled magnetic doublet. ${ }^{[42]}$ These microvortices could induce a fluidic trap, which enables the capture of microobjects provided that the rotation frequency is high enough. Because the microvortices are localized nearby the center of the rotating microactuator and decay rapidly as the distance increases, they function as a type of highly focused fluidic tweezers (hereafter referred to as FT) attached to the microactuator. The position of the FT can be precisely controlled to selectively trap and transport individual microobject with the presence of an underlying solid surface, which enables the microactuators to break the symmetry of motion and generate net propulsion in the low Reynolds number regime. ${ }^{[43-45]}$ By switching the rotation plane of the microactuator through tuning the rotating magnetic fields, both translational (for transport of microobjects) and static (for immobilization of microobjects) control can be realized.

A limitation of the NW FT is that its trapping force is relatively small, and effective trapping of heavy microobjects, if still capable, requires high rotation frequencies of the magnetic field. This may have several adverse effects. First, Ni NWs of different length are characterized with their own step-out frequencies, ${ }^{[46]}$ namely the maximum synchronized frequency for a certain NW under a magnetic field of certain strength. If the operating frequency required for this NW to induce fluidic trapping is higher than its step-out frequency, an increasing demand of the field strength arises, which would practically lower the stability of the electromagnetic coils due to potential overheating. Second, the impedance of the electromagnetic coils used to generate the magnetic field can increase too much due to coil 
induction at high frequency, and hence a higher voltage needs to be provided to maintain certain field strength. Again, this may contribute to over-heating of the magnetic setup and render the system unable to work continually. Third, synchronization of the magnetic field with fast-varying current input may be considerably delayed due to the coils' induction effect at high frequencies. This can affect the stability of the electromagnetic system and consequently the accuracy of the FT's locomotion, eventually impairing the FT's capability of implementing cargo transport with high precision. Aimed at enhancing the efficacy of fluidic trapping as well as its versatility to implement on-demand manipulation, a DB FT is developed in this work. Apart from the tumbling motion (rotating about the short axis) presented by a $\mathrm{Ni} \mathrm{NW}$, the $\mathrm{DB}$ microactuator is also able to perform wobbling and rolling motion, for which effective fluidic traps can also be induced. Benefiting from the combination of fluidic traps induced by the three motions, more delicate manipulation can be realized.

\section{Experiments}

\subsection{Fabrication and control of magnetic DB}

To fabricate a magnetic $\mathrm{DB}$, a Ni NW was manipulated with a rotating magnetic field to introduce its both ends into tiny holes on $6 \mu \mathrm{m}$-diameter polystyrene (PS) microbeads which have similar density to liquid water (Figure 1a, b, c and Supporting Information Video V1). The assembled DBs in general show good stability and dissociation of the microbead from the DB body is very rare (only observed for several samples when the rotation direction of the magnetic field was abruptly changed under high frequency). We believe that the firm junction is due to a strong adhesive force between the NW and the microbead. A shortcoming of the current process is that the assembly is random in a certain sense and a success may request several trials. In future work, we plan to fabricate DBs of controllable size in a systematic manner by using direct laser writing

combining physical vapor deposition, the former technique producing a designated 
shape and the latter magnetizing it. Standard control of the DB relies on its tumbling motion, i.e., rotation about its minor axis. By tuning the rotation plane with the rotating magnetic field, both translational and static controls can be realized, which are for the transport and immobilization of microobjects, respectively. In translational control, the normal of the rotation plane is parallel to the solid wall. The DB will experience distinct drag resistance on its two ends when tumbling near a solid surface. This results in a net displacement of the DB for a full rotation cycle, thus enabling translation along the wall while rotating above it (Figure 1d). In static control, the normal of the ration plane is perpendicular to the boundary, and the DB will spin locally without translation (Figure 1e). Shown in Figure 1f is a mode unique to DB and not available to NW, namely the rolling motion. This mode is of particular interest to us, and will be elaborated later.

Actuated by a uniform magnetic field from Helmholtz coils, the DB would only experience magnetic torque, but no force. Hence, in the low Reynolds number regime (both the translational and rotational Reynolds number of the rotating DB under our experimental conditions are by far smaller than order unity), the DB's motion in DI water merely relies on the instantaneous magnetic torque caused by misalignment. In principle, a magnetic body will automatically align its axis of magnetization (normally the long dimension of the body) with the direction of the external magnetic field due to the torque. To rotate the body, this misalignment must be maintained through continuously rotating the magnetic field. The magnetic torque $\overrightarrow{T_{m}}$ exerted by an external field $\overrightarrow{B_{m}}$ on a magnetic body of volume $V$ is calculated as $\overrightarrow{T_{m}}=V \vec{M} \times \overrightarrow{B_{m}}$, where $\vec{M}$ stands for the magnetization of the magnetic material. In experiments, the magnetization direction of a Ni NW always coincides with its long axis, which leads to tumbling rotation when the NW is exposed to an external magnetic field. For a magnetic DB in our experiments, however, the apparent magnetization is found more complex. Its direction varies depending on the frequency and strength of the applied magnetic field, thus resulting in different motion regimes, e.g. tumbling, wobbling, and rolling, as will 
be demonstrated by our experimental results. This complexity of the apparent magnetization for DB is presumably caused by certain asymmetry of the connection between the NW and microspheres. Given the random nature of the assembly process, the alignment of the NW is unlikely to coincide with the center-center axis of the DB. Further, because the NW is the only magnetic part of the dumbbell, this asymmetric alignment will result in a magnetic moment that can be decomposed into both transverse and longitudinal directions of the DB. Previously, the underlying physics for an elongated magnetic body to perform dynamical motion when exposed to magnetic field has been studied both experimentally and theoretically by Ghosh et al., ${ }^{[47]}$ and Morozov and Leshansky. ${ }^{[48]}$ They considered a helical micro/nanoscale magnetic body whose magnetization is pointing somewhere between its short and long axes, suggesting magnetic moment components for actuation in both directions. Due to distinct drag coefficients in the transverse and longitudinal directions, the orientation of the helix will change dynamically to minimize viscous dissipation subject to varying magnetic fields. Quantitatively, the dynamics depends on both the magnetization direction and shape anisotropy of the magnetic body.

As shown in the supplementary videos (Supporting Information Videos V2-3), rather than simply tumbling when exposed to magnetic field as a NW does, the DB is able to perform three-modal motion: rolling (0-1 mT), wobbling (1-5 mT) and tumbling ( $\geq 5 \mathrm{mT})$, for which the precession angle increases in turn. Furthermore, real-time transition between the three modes can be realized simply by adjusting the input strength of the rotating magnetic field without changing the rotation frequency or rotation axis. This unique property of DB enables a new approach of trapping objects, namely rolling trapping, which will be elaborated later in the text. Figure $1 \mathrm{~g}$ characterizes the dynamical behavior of two different DBs under a rotating magnetic field with constant rotation frequency but varying strength. DBs of similar size will be presented in the following cargo loading and transport 
experiments, where trapping capability under all motion regimes was observed. Note that in previous works, ${ }^{[47,48]}$ the dynamics were achieved by changing the frequencies of the magnetic field while keeping the field strength constant, whereas in our case the frequency is kept constant and we vary the field strength instead. Ghosh et al. observed tumbling, procession (equivalent to wobbling) and propulsion (equivalent to rolling) of the helix in turn as the frequency was gradually increased. ${ }^{[47]} \mathrm{By}$ increasing the magnetic field strength, the order is reversed, i.e., first rolling, then wobbling, and last tumbling. We also carried out an experiment using the DB in which the frequency was increased from $1 \mathrm{~Hz}$ to $30 \mathrm{~Hz}$ while keeping the field strength as $4 \mathrm{mT}$. Then the dynamics resembling Ghosh et al. ${ }^{[47]}$ was observed: the DB initially takes on tumbling motion within 1-20 Hz; further increase of the frequency leads to wobbling motion until $28 \mathrm{~Hz}$, beyond which the DB assumes a rolling regime. To a physicist's view, the two transitions, namely under a magnetic field of varying frequency and varying strength are essentially the same problem and can be unified by constructing a dimensionless number for the magnetic field.

\subsection{Cargo loading and transport}

Experiments of cargo loading and transport were carried out using identical polystyrene microbeads that have been used to assemble the DB actuator. The rotational flows induced by the moving DB are found to be highly localized, even with the perturbing tumbling motion. Since the locomotion of the microacuator can be promptly controlled through manipulating the rotation axis and frequency of the magnetic field, selective trapping and transport of individual microobjects can be realized. Experimental results show that when the tumbling DB with or without a trapped microbead passes by stationary microbeads that are randomly distributed on the solid surface, no significant displacement of them is observed (left panel of Figure 2 and Supporting Information Video V4). With a $5 \mathrm{mT}$ magnetic field rotating at 20 
$\mathrm{Hz}$, a translation speed of $76 \mu \mathrm{m} / \mathrm{s}$ can be achieved for the DB to approach the microbead. The value will be slightly decreased to $72 \mu \mathrm{m} / \mathrm{s}$ after it traps the target. Note that the speed of this DB is different from the value measured for a similar DB under the same magnetic field in Figure 1f. This variance of speed may have been caused by the difference of fluid viscosity, which can be affected by the concentration of ethanol in the solution that have been used to disperse the Ni NW and PS microbeads.

Once trapped, the microbead travels with the DB at the same speed. After reaching the destination, immobilization is realized by tilting the rotation axis of the magnetic field by $90^{\circ}$. The DB is then under static control and will only spin locally. Since the rotation plane now coincides with the observing plane of the microscope, fluid streamlines nearby the rotating DB as well as the trajectory of the trapped microbead can be examined. As the right panel of Figure 2 shows, a microvortex is formed above the rotating DB, whose streamlines resemble that of a "streaming cell" reported in experiments of steady streaming. ${ }^{[35-39]}$ Under low or moderate rotation frequencies, the trapped microbead follows the fluid trajectory and orbits the microvortex center with self-spinning motion. By abruptly increasing the rotation frequency of the magnetic field to frequencies above $20 \mathrm{~Hz}$, the microbead would deviate from the fluid trajectory, spiral across the streamlines, and migrate inward until firmly trapped in the center, leading to the so-called fluidic trapping. The mechanism of this fluidic trapping is still physically unclear, and discussion on it will be presented later.

Despite the robust trapping capability of DB in the tumbling mode, there are also some side effects with it for cargo loading. First, the DB translates much faster than a NW, and the repulsive shear flow arising from the motion is also stronger; consequently, the DB could pass by quickly or the microbead could be pushed away abruptly, before fluidic trapping occurs. Second, the location of microvortices induced above the DB can be several microns away the solid surface given the dimension of its sphere, thus producing a gap between the fluidic trap and objects lying on the surface. Nevertheless, attributed to the DB's dynamics, a premium 
strategy is possible to load microobjects smoothly: to initially approach the target in rolling mode and trap them laterally (Supporting Information Videos V5-6). When the DB is translating in the rolling mode, microvortices are generated nearby its both ends, which can be harnessed to trap objects. Because the rolling DB can retain high rotation frequency while performing a low/moderate translational speed, it can gently approach the target and capture it with a strong fluidic trap. After the target is successfully trapped, the DB can easily resume tumbling motion for speedy transport without losing the target, simply by promptly increasing the input field strength (Figure 3).

Another noteworthy finding about the rolling mode is that the DB can stably trap two microobjects on both sides. Indeed, for all the three motion regimes, two symmetric microvortices and thus equivalent fluidic traps can be formed. However, for tumbling and wobbling regimes, after one object is trapped the microvortex on the other side seems disrupted and that fluidic trap ceases to function. This disruption is avoided in the rolling regime as the two fluidic traps are relatively distant and do not interfere with each other. Note that wobbling trapping can either be regarded as a transient state between rolling trapping and tumbling trapping or a degraded form of the latter, so we skip further discussion about it here. The controllable transition between these three trapping scheme is desirable in two respects. On the one hand, it suggests more versatile micromanipulation for on-demand tasks, e.g., combination of mild cargo loading and speedy cargo transport. On the other, it implies more flexible cargo transport in confined geometries considering that the DB FT can adjust its orientation as needed and perform dynamical locomotion.

\subsection{Cargo release}

Cargo release for DB FT is implemented in two steps. The first step is to switch the DB from translational control to static control when the targeted position is arrived at. This transition is 
achieved by altering the rotation axis of the magnetic field so that the rotation plane of the DB changes from vertical to horizontal. Petit et al. showed that the trapped microbead by NW FT in spinning manner (i.e., rotating locally) can then be released by abruptly tilting the NW by $90^{\circ}$ again at a relatively low rotation frequency and further reducing the amplitude of the microvortex through lowing the rotation frequency subsequently. ${ }^{[42]}$ This strategy of cargo unloading, however, does not work well for DB FT as the second step, because even at low frequencies, the microbead can still be trapped by or attached to the DB. Alternatively, we make use of the dynamics of the DB. Firstly, by decreasing the magnetic field strength, the locomotion of the DB is gradually changed from tumbling to wobbling, and further, rolling. Then, by tilting the rotation plane by $90^{\circ}$, the DB rotates like a whirligig. Meanwhile, the microbead leaves the original fluidic trap and rotates around the DB. Next, by increasing the magnetic field strength, the microbead will be pushed away from the center of the DB FT. Now the DB is free and can tumble away without causing significant disturbance to the microbead (Supporting Information Video V7).

\section{Simulations}

\subsection{Comparison of induced tangential flows}

To understand the enhanced trapping capability of DB shown by the experiments, a finite element method (FEM) model is established using the Rotating Machinery module of COMSOL Multiphysics ${ }^{\circledR}$ to investigate the induced fluid flows by the spinning motion of both DB and NW in liquid water (Figure 4a and Supporting Information Figure S1). The NW is modeled as a tiny cylindrical body of $8 \mu \mathrm{m}$ in length and $200 \mathrm{~nm}$ in diameter. To make the DB comparable, it is modeled as the assembly of an identical NW and two $6 \mu \mathrm{m}$-diameter microspheres, whose centers coincide with the NW's two ends. Simulation results reveal that for the tangential flow induced in the rotation plane of the DB rotating at $20 \mathrm{~Hz}$, maximal velocities $(879.6 \mu \mathrm{m} / \mathrm{s})$ occur at two ends and a local minimum (zero) at the center (Figure 
4b). After averaging over one full rotation, radial symmetry of the velocity is expected, suggesting concentrated microvortices similar to those reported by Petit et al. for a rotating NW. ${ }^{[42]}$ The existence of microvortices is further verified by the finding of significant vorticity in the central area of the DB (inset, Figure 4c), which was previously analyzed by researchers to be crucial for the cross-streamline spiral trajectory of inertial particles in the rotational flow. ${ }^{[49]}$

From Figure 4c, we can clearly observe a nest-like spatial confinement constructed by the deformed streamlines above the DB center, visually serving as a "container" of microobjects. For a rotating NW, the streamlines are relatively planar, and no such threedimensional confinement can be found (Figure 4e). An intuitive conjecture is that this confining effect of DB contributes to a more effective and stable fluidic trap. Compared with a NW, the introduction of microspheres at both ends for the DB significantly amplifies the strength of vorticity in the central region above the rotating body. Specifically, an amplification factor about 3 to 4 can be found for the peak vorticity in the plane $1 \mu \mathrm{m}$ above the rotation plane (insets of Figure $4 \mathbf{c}$ and $4 \mathbf{e}$ ). Note that asymmetry of streamlines about the rotation plane is caused by the presence of a no-slip wall underneath the rotating body. For ideal rotation in unbound fluid without this wall, streamlines near the rotating body would be symmetric about the rotation plane (Figure 4f), which implies identical microvortices on both sides. With the addition of a solid wall near the rotating body, the streamlines are bent towards the opposite side of the wall (Figure 4d), thus notably altering the flow morphology and enhancing the spatial confinement on the upper side. Meanwhile, the strength of the microvortices on the same side are found increased, exemplified by the comparison of zcomponent vorticity for the two cases in a plane $1 \mu \mathrm{m}$ above the rotation plane (Figure $4 \mathbf{d}$ and 4 f).

To gain insight into this amplification of vorticity, we investigate the tangential flows induced nearby a DB rotating at $20 \mathrm{~Hz}$ and a NW rotating at $95 \mathrm{~Hz}$, the chosen frequencies 
being the critical values when fluidic trapping occurs for the DB and NW, respectively. While the magnitude of tangential flow vanishes rapidly for NW as the distance from the rotation plane increases (Figure 5b), notable tangential flow still exists for DB even at a distance of 6 $\mu \mathrm{m}$ away (Figure 5a). The question now is: how does this flow pattern contributes to trapping the microobject? Chong et al. suggested that the trapping intensity of a streaming cell would increase with larger velocity gradients, ${ }^{[49]}$ the first order of which being shear rate, and the second being vorticity. Given the hydrodynamic similarity of the streaming cell and our fluidic trap, we hypothesize that the velocity gradients are also important indicators for fluidic trapping in our case. In the last paragraph, the increase of vorticity strength for DB has been demonstrated. Next, we proceed to analyze the magnitude of shear rates. Since the shear rates of utmost interest are in regions of where microobjects are initially captured by the flow, we focus only on a range of distance $2 \mu \mathrm{m}$ to $6 \mu \mathrm{m}$ away from the rotating body.

As Figure 5c shows, despite a much lower rotation frequency of $20 \mathrm{~Hz}$, the overall magnitude of shear rates arising from the DB's tangential flows is still larger than a NW rotating at $95 \mathrm{~Hz}$. For the $\mathrm{NW}$, shear rates at $6 \mu \mathrm{m}$ from the rotation plane is nearly negligible, but for the DB, the shear rates there are still considerable. Furthermore, the DB's shear rates present a distinctive pattern: high shear rates arising from the DB's rotation are mainly concentrated nearby the outer periphery of the sphere, while the magnitudes in the region of interest, i.e., the central area where the trapped microobject will stably reside, are significantly smaller. This distribution of shear rates is rather beneficial for the trapping of biological samples. It induces strong fluidic forces possessed by the DB to initiate trapping when approaching a microobject, yet low shear stress and thus low possibility of hydrodynamic damage to the target once it is trapped. More discussion on the shear stress regarding the manipulation of biological samples can be found at the end of the article. 


\subsection{Analysis of rolling trapping}

The rolling mode of the DB discovered in our experiments is of particular interest because it can serve as a brand new trapping technique that largely facilitates the manipulation of microobjects. In this mode, the dumbbell rotates about its long axis, and translates with a moderate velocity above the solid surface in a direction perpendicular to the axis. While advancing, microvortices are generated at both ends of the DB, i.e., outer periphery of the PS microbead, which can trap microobjects nearby the DB's path in a gentle but effective manner. We illustrate this trapping scheme using a single sphere vertically rotating above a no-slip wall. As reported by Ye and Sitti, ${ }^{[17]}$ rotational flows in the horizontal plane generated by an upright spinning magnetic microsphere induce a fluid force that can capture motile microorganisms. By tilting the rotation axis, the microsphere translates on a glass substrate through rolling while retaining the fluidic force for trapping. This enables transportation of the microorganism. However, when the rotation axis is fully tilted to be parallel to the substrate, i.e., pure rolling motion as in our case, only translation is presented by Ye and Sitti, because the rotational flows considered by them are no longer available for trapping. Here, we explore another possibility to trap microobjects in this configuration: using vertical microvortices on the lateral side of the spinning sphere. To visualize this trapping scheme, we carried out a simulation for a $5 \mu \mathrm{m}$ sphere rotating at $100 \mathrm{~Hz}$, the same as simulated by Ye and Sitti, in COMSOL Multiphysics. For simplicity, we only consider the sphere to be locally spinning.

Simulation results of the rotational flow in the equatorial plane of the spinning microsphere agree well with the finding by Ye and Sitti: the flow presents circular streamlines with the magnitude of velocity decaying rapidly as the distance from the sphere surface increases, and a maximum value of $1571 \mu \mathrm{m} / \mathrm{s}$ is found on the surface (Figure 5d). However, the velocity near the top of the sphere (i.e., its lateral side), where the flow resembles a streaming cell (Figure 5e), has a distinct pattern of velocity magnitudes (Figure 5f). With a 
minimum occurring at the center and two maxima at $0.75 a$ ( $a$ is the radius of the sphere), the velocity will lead to a microvortex reminiscent of the one reported by Petit et al. after averaged over a full rotation. ${ }^{[42]}$ In the rolling mode of our DB, the rotation axis is titled to be parallel to the wall, and two such microvortices will appear on lateral sides of its both spheres. Although the morphology and strength of the microvortices are subject to further change due to wall effect (causing complex deformation of streamlines nearby), its capability to trap microobjects is still robust as has been proven by our experiments.

\section{Discussion}

\subsection{Effectiveness of magnetic actuation}

We also experimentally characterize the translation velocity of the DB against rotation frequency in comparison with a NW of equivalent length (Figure 6a). Except for certain fluctuating data points, significant enhancement of mobility is found. With a rotating magnetic field of the same strength, the translation speed of the DB can be five times as high as the NW's (e.g., at $15 \mathrm{~Hz}$ or $32 \mathrm{~Hz}$ ). In terms of fluidic trapping, a much lower threshold frequency $(20 \mathrm{~Hz})$ is required for DB FT than NW FT $(95 \mathrm{~Hz})$ as shown in the experiments. This has multiple implications. First, constrained by a constant magnetic field strength, the frequency range within which fluidic trapping can be realized is wider for the $\mathrm{DB}$, assuming that it has a similar step-out frequency to a NW of equivalent length. In other words, the DB FT is more flexible and suitable to manipulate microobjects of varying weights (i.e., lighter or heavier). Second, the heating effect of the electromagnetic system can be largely relieved. The coils that compose the magnetic setup are inductors, and their impedance increases with the rotation frequency of the magnetic field. Since the operating frequency for DB FT is significantly decreased, the working voltage is also smaller, thus reducing the Joule heat of the coils and extending the operation time of the setup within bearable temperature load. Third, potential delay of the magnetic field's synchronization with fast-varying current input 
due to the coils' induction effect at high frequency is also circumvented, which means the microactuator's locomotion can be more promptly and hence precisely controlled.

\subsection{Efficiency of cargo transport}

To evaluate the efficacy of cargo transport for DB FT, we look into the velocity loss due to cargo loading. The case presented in Figure 2 (left panel), in which the DB FT is tumbling under a $5 \mathrm{mT}$ magnetic field with a rotation frequency of $20 \mathrm{~Hz}$, is examined. The transitional velocity of the tumbling DB before loading is measured to be $76 \mu \mathrm{m} / \mathrm{s}$. After loading, the DB translates together with the trapped microbead at a velocity of $72 \mu \mathrm{m} / \mathrm{s}$. In this case, the velocity loss is only about $5 \%$, which is decreased by a factor of $2-3$ in comparison with that of the NW FT also in tumbling motion (Figure 6b). This indicates that DB FT is a more efficient tool for cargo transport. Furthermore, DB FT's feasibility to trap microobjects of different size or number is also expected to be better, considering the $3 \mathrm{D}$ confinement and trapping focus of the fluidic trap can be more effectively optimized via changing the wire length and bead size in coordination.

\subsection{Mechanism of fluidic trapping}

The physical origin of the fluidic trapping by mobile microvortices at low Reynolds number is still analytically unresolved. But given its similarity in both flow structure and particle behavior to viscous streaming trapping inertial particles, the two trapping mechanisms may have some physical reasoning in common. For the latter, diversion of the trapped particle's trajectory from the fluid streamline has been theoretically analyzed by Chong et al. to be caused by an inward hydrodynamic force in flow regions of high vorticity. ${ }^{[49]}$ This hydrodynamic force, or interpreted as trapping force, originates from the dominant effect of the integrated Faxén terms (representing the impact of non-uniform fluid velocity incident upon inertial particles) and Saffman lift, ${ }^{[50,51]}$ countering the Stokes drag, the convective term

and the Basset history term in the modified Maxey-Riley equation (the governing equation of 
a small rigid sphere's motion in non-uniform flows). ${ }^{[52]}$ To be specific, the Faxén terms and Saffman lift contribute to a non-zero relative velocity of the inertial particle from the fluid particle, while the others tend to drive the relative velocity back to zero. The inertial particle would eventually be trapped at the center the streaming cell, which is an equilibrium position where all the forces are balanced.

Based on the simulation results of tangential velocity and shear rate, both the particle slip Reynolds number and shear Reynolds number are calculated to be much smaller than the order of unity, which are prerequisites for us to invoke the modified Maxey-Riley equation with Saffman lift involved. The particle slip Reynolds number is expressed as $R e_{\text {slip }}=$ $a|\vec{w}| / \nu$, and the shear Reynolds number $R e_{\text {shear }}=a^{2} G / v$, where $a, \vec{w}, v$ and $G$ represent the microbead's radius, relative velocity to the fluid particle (normally smaller than the undisturbed fluid velocity at the center of the particle), kinematic viscosity and shear rate in the direction of relative motion, respectively. According to the simulation results, at a distance of $3 \mu \mathrm{m}$ away from the DB's rotation plane, the maximum tangential velocity and local shear rate the microbead can encounter is about $560 \mu \mathrm{m} / \mathrm{s}$ and $170 \mathrm{~s}^{-1}$, respectively. With $a=$ $3 \mu \mathrm{m}$ and $v=1.0 \times 10^{-6} \mathrm{~m}^{2} / \mathrm{s}$, we assume that $|\vec{w}|$ and $G$ to be of the same order to the tangential velocity and local shear rate, i.e., $O\left(10^{-4}\right)$ and $O\left(10^{2}\right)$, then $R e_{\text {slip }}$ and $R e_{\text {shear }}$ are calculated to be of only $O\left(10^{-4}\right)$ and $O\left(10^{-3}\right)$, respectively.

To validate the analysis relying on the Maxey-Riley equation for our PS microbead's motion in the flow, we need to make sure that rotational flows of the induced microvortices by the rotating DB are only locally modified, but not severely disturbed by the microbead's motion. Given that the PS microbead has a similar (slightly larger) density to the fluid particle (DI-water), also that the rotational flows are quite strong as revealed in the simulation section, we assume that the trapping system satisfied the above criterion. This assumption is further justified by a trapping experiment shown in Figure 7. In the figure, apart from a microbead which is already immobilized by the fluidic trap at the microvortex center, there exist another 
microbead of the same size and a yeast cell of much smaller size captured in the rotational flows of the microvortex. It can be clearly observed that, the yeast cell in the orbit (vertically standing) nearer to the microvortex center moves much faster than the microbead in a larger orbit. Initially it is at the same azimuthal position with the microbead, but soon found chasing the latter. After succeeding in the pursuit, its motion is disturbed by local flows induced by the microbead and deviates from the original orbit. It is then "detained" by the microbead for a while, yet in the end separated again from the microbead. Eventually, it falls in the same orbit with the microbead, and travels with a slower speed than the latter. Refer to Table $\mathbf{1}$ for the azimuthal positions and instantaneous angular speeds of the microbead and yeast at the extracted time points. The results of this experiment imply that the rotational flow induced by the rotating DB microactuator is of predominant effect, and the secondary flow arising from the microbead's motion only plays a limited role.

\subsection{Trapping force and shear stress}

A primary interest of this fluidic trapping harnessed by both DB and NW fluidic tweezers is its trapping force. Experimentally, an estimate of this trapping force can be attained by measuring the mobility force of microorganisms upon escaping from this fluidic trapping, which has been applied to measure the trapping force of hydrodynamic tweezers. ${ }^{[39]}$ To be more accurate, laser tweezers or electrostatic devices need to be employed to measure the force, which could be a direction of future work. For a rough estimate of the trapping force's magnitude, we can look at the viscous drag force experienced by the trapped microbead. During cargo transport, the microbead is firmly trapped in the microvortex and translate with the DB at the same speed. We assume that the trapping force exerted on the microbead by the DB FT is larger than the drag force by the fluid. To calculate this drag force, the Stokes' law for a small sphere moving at low Reynolds number can be applied, i.e., $F_{d}=6 \pi \eta a V$, where $\eta, a$ and $V$ are the fluid viscosity, sphere radius and 
relative velocity, respectively. We take the microbead in Figure 2 for example, where $\eta=1.0 \times 10^{-3} P a \cdot s$ (DI water), $a=3 \mu m$ and $V=72 \mu m / s$, resulting in a drag force $F_{d}=4.07 p N$. This magnitude serves as a lower bound estimate for the trapping force, as the microbead can still be firmly trapping at higher velocities.

For the manipulation of biological samples, shear stress is also critical. Excessive shear stress may exert too much pressure on the membrane-fluid interface of biological samples, and consequently cause shear-induced detachment or even fatal damage. Based on the simulation results, local shear stress near the FT can be calculated as product of the shear rate and fluid viscosity. At the frequency upon DB fluidic trapping, namely $20 \mathrm{~Hz}$, the largest shear rate $1 \mu \mathrm{m}$ away from the DB reads $286.5 \mathrm{~s}^{-1}$, indicating a shear stress of $0.2865 \mathrm{~Pa}$ in the case of DI water (i.e., $\eta=1.0 \times 10^{-3} \mathrm{~Pa} \cdot s$ ). Given the normal physiologic levels of shear stress within arteries, i.e., 1-7 $\mathrm{Pa},{ }^{[53]}$ the shear stress imposed on microobjects trapped by the DB FT should be biologically safe. Furthermore, note that this maximum shear stress is estimated based on an inertial particle without relative velocity with the fluid particle, while during fluidic trapping the trapped microobject will be in continual motion relative to the fluid, spiralling across the circular streamlines of the induced flow. And when it eventually arrives at the vortex center and remains stably trapped, the magnitude of shear rate there becomes relatively small. Taking all these into account, the actual shear stress experienced by the trapped microobjects during the trapping process should be even smaller than the estimated value above. Last but not least, owing to the availability of premium trapping scheme, i.e., rolling trapping, the possibility of biological damage is further lowered.

\section{Conclusion}

In this work, a dumbbell (DB) microactuator is developed as a type of versatile fluidic tweezers (FT) to manipulate individual microobjects using a low-strength rotating magnetic field. Our experiments demonstrate stronger trapping capability and cargo-transport 
performance of the DB FT in comparison with NW FT. And simulations further investigate the reasons for the enhancement in terms of flow features nearby the microactuators. Equipped with the capability of dynamical trapping in three modes, DB FT can provide more versatile manipulation of microobjects. Also, the translation of DB FT is much faster under the same magnetic field, and the velocity loss due to cargo transport is much smaller. At last, the shear stress exerted on trapped microobjects is addressed, which is well within physiological levels due to the conducive flow pattern and flexible trapping strategy of DB FT. With all these desirable characteristics, we conclude that DB FT offers a promising microrobotic tool for micromanipulation of biological samples. For future work, we plan to employ direct laser writing and physical vapor deposition to fabricate DB microactuators of a range of sizes. Then systematic characterization of the DB FT's mobility and trapping capacity under different operating conditions (e.g., frequency/strength of the magnetic field, viscosity of the biofluidic medium, dimension of the target microobject) will be conducted for practical applications.

\section{Experimental section}

Materials: Nickel (Ni) nanowires (NWs) were synthesized using a template-assisted electrochemical deposition method in an anodic aluminium oxide (AAO) template. Polystyrene (PS) microbeads of $6 \mu \mathrm{m}$ in diameter were purchased from Polyscience, Inc (Polybead®).

Fabrication of dumbbell microactuator: The dumbbell (DB) structures were assembly using the Ni NWs and PS microbeads as described above. The method of assembly has been stated in the main text, and the process is presented in Supporting Information Video V1.

Cargo loading, transport, and release: These experiments were conducted in deionized (DI) water contained in an acrylic tank $(L \times W \times H=15 \mathrm{~mm} \times 15 \mathrm{~mm} \times 5 \mathrm{~mm})$, which was placed via a micropositioner, namely a linear translation stage, at the center of a 
customer-made three-axial Helmholtz-coil setup equipped with a PC controller and a vision system. The experimental setup is shown in Supporting Information Figure S2. Major components include: servo amplifier (× 3 , Maxon ADS-50@Switzerland), power supply (RS Pro@Taiwan), analog/digital I/O board (Sensoray model-626@US), CCD (Point Grey Flea2@Canada), microscope (Correct, Seiwa Optical@US), Helmholtz coils ( $\times 3$, designed and fabricated by the authors), linear translation stage (Newport M-460A@UK).

\section{Supporting Information}

Supporting information is available from the Wiley Online Library or from the author.

\section{Acknowledgements}

This work was financially supported by the Early Career Scheme (ECS) grant with Project No. 439113, the General Research Fund (GRF) with Project No. 14209514, 14203715 from the Research Grants Council (RGC) of Hong Kong SAR, the National Natural Science Funds of China for Young Scholar with the Project No. 61305124, the grant from Shenzhen Government (SZSTI) for the Basic Research Fund with Project No. JCYJ20140905151415999 and the National Research Foundation of Korea (NRF) from the Ministry of Science, ICT \& Future Planning with Project No. 2014R1A2A2A01006223. The authors would also like to thank the support from the CUHK T Stone Robotics Institute.

\section{References}

[1] L. Zhang, J. J. Abbott, L. Dong, B. E. Kratochvil, D. Bell, B. J. Nelson, Appl. Phys. Lett. 2009, 94, 64107.

[2] B. J. Nelson, I. K. Kaliakatsos, J. J. Abbott, Annu. Rev. Biomed. Eng. 2010, 12, 55.

[3] K. E. Peyer, L. Zhang, B. J. Nelson, Nanoscale 2013, 5, 1259.

[4] K. E. Peyer, S. Tottori, F. Qiu, L. Zhang, B. J. Nelson, Chem. - Eur. J. 2013, 19, 28.

[5] E. Diller, M. Sitti, Found. Trends ${ }^{\circledR}$ Robot. 2013, 2, 143.

[6] M. Sitti, H. Ceylan, W. Hu, J. Giltinan, M. Turan, S. Yim, E. Diller, Proc. IEEE 2015, 103, 205.

[7] L. Zhang, T. Petit, Y. Lu, B. E. Kratochvil, K. E. Peyer, R. Pei, J. Lou, B. J. Nelson, $A C S$ Nano 2010, 4, 6228. 
[8] C. Hu, C. Tercero, S. Ikeda, T. Fukuda, F. Arai, M. Negoro, In 2011 IEEE/RSJ International Conference on Intelligent Robots and Systems (IROS); 2011; pp. 439-444.

[9] S. Tottori, L. Zhang, F. Qiu, K. K. Krawczyk, A. Franco-Obregón, B. J. Nelson, Adv. Mater. 2012, 24, 811 .

[10] T. S. Anirudhan, S. Sandeep, J. Mater. Chem. 2012, 22, 12888.

[11] K. M. Sivaraman, C. Kellenberger, S. Pané, O. Ergeneman, T. Lühmann, N. A. Luechinger, H. Hall, W. J. Stark, B. J. Nelson, Biomed. Microdevices 2012, 14, 603.

[12] L. Zhang, T. Petit, K. E. Peyer, B. J. Nelson, Nanomedicine Nanotechnol. Biol. Med. 2012, 8, 1074.

[13] S. Kim, F. Qiu, S. Kim, A. Ghanbari, C. Moon, L. Zhang, B. J. Nelson, H. Choi, Adv. Mater. 2013, 25, 5863.

[14] S. Floyd, C. Pawashe, M. Sitti, IEEE Trans. Robot. 2009, 25, 1332.

[15] K. E. Peyer, L. Zhang, B. J. Nelson, Appl. Phys. Lett. 2011, 99, 174101.

[16] Z. Ye, E. Diller, M. Sitti, J. Appl. Phys. 2012, 112, 64912.

[17] Z. Ye, M. Sitti, Lab. Chip 2014, 14, 2177.

[18] T.-Y. Huang, F. Qiu, H.-W. Tung, X.-B. Chen, B. J. Nelson, M. S. Sakar, Appl. Phys. Lett. 2014, 105, 114102.

[19] J. Zheng, X. Xing, J. Evans, S. He, NPG Asia Mater. 2016, 8, e257.

[20] A. Ashkin, J. M. Dziedzic, J. E. Bjorkholm, S. Chu, Opt. Lett. 1986, 11, 288.

[21] D. G. Grier, Nature 2003, 424, 810.

[22] P. Y. Chiou, A. T. Ohta, M. C. Wu, Nature 2005, 436, 370.

[23] F. H. C. Crick, A. F. W. Hughes, Exp. Cell Res. 1950, 1, 37.

[24] N. Wang, J. P. Butler, D. E. Ingber, Science 1993, 260, 1124.

[25] C. Gosse, V. Croquette, Biophys. J. 2002, 82, 3314.

[26] H. A. Pohl, Dielectrophoresis: the behavior of neutral matter in nonuniform electric fields; Cambridge University Press: Cambridge; New York, 1978.

[27] J. P. Hoogenboom, D. L. J. Vossen, C. Faivre-Moskalenko, M. Dogterom, A. van Blaaderen, Appl. Phys. Lett. 2002, 80, 4828.

[28] D. Erickson, X. Serey, Y.-F. Chen, S. Mandal, Lab. Chip 2011, 11, 995.

[29] M. Righini, P. Ghenuche, S. Cherukulappurath, V. Myroshnychenko, F. J. García de Abajo, R. Quidant, Nano Lett. 2009, 9, 3387.

[30] J. C. Ndukaife, A. V. Kildishev, A. G. A. Nnanna, V. M. Shalaev, S. T. Wereley, A. Boltasseva, Nat. Nanotechnol. 2016, 11, 53.

[31] M. Krishnan, N. Mojarad, P. Kukura, V. Sandoghdar, Nature 2010, 467, 692.

[32] A. P. Fields, A. E. Cohen, Proc. Natl. Acad. Sci. 2011, 108, 8937.

[33] Q. Wang, W. E. Moerner, ACS Nano 2011, 5, 5792.

[34] J. Shi, X. Mao, D. Ahmed, A. Colletti, T. J. Huang, Lab. Chip 2008, 8, 221.

[35] B. R. Lutz, J. Chen, D. T. Schwartz, Proc. Natl. Acad. Sci. 2003, 100, 4395.

[36] B. R. Lutz, J. Chen, D. T. Schwartz, Phys. Fluids 1994-Present 2005, 17, 23601.

[37] B. R. Lutz, J. Chen, D. T. Schwartz, Anal. Chem. 2006, 78, 1606.

[38] V. H. Lieu, T. A. House, D. T. Schwartz, Anal. Chem. 2012, 84, 1963.

[39] B. R. Lutz, J. Chen, D. T. Schwartz, Anal. Chem. 2006, 78, 5429.

[40] C. M. Lin, Y. S. Lai, H. P. Liu, C. Y. Chen, A. M. Wo, Anal. Chem. 2008, 80, 8937.

[41] T. Hayakawa, S. Sakuma, F. Arai, Microsyst. Nanoeng. 2015, 1, 15001.

[42] T. Petit, L. Zhang, K. E. Peyer, B. E. Kratochvil, B. J. Nelson, Nano Lett. 2012, 12, 156.

[43] E. M. Purcell, Am. J. Phys. 1977, 45, 3.

[44] E. Lauga, T. R. Powers, Rep. Prog. Phys. 2009, 72, 96601.

[45] J. J. Abbott, M. C. Lagomarsino, L. Zhang, L. Dong, B. J. Nelson, Int. J. Robot. Res. 2009.

[46] L. Zhang, J. J. Abbott, L. Dong, K. E. Peyer, B. E. Kratochvil, H. Zhang, C. Bergeles, B. J. Nelson, Nano Lett. 2009, 9, 3663.

[47] A. Ghosh, P. Mandal, S. Karmakar, A. Ghosh, Phys. Chem. Chem. Phys. 2013, 15, 10817. 
[48] K. I. Morozov, A. M. Leshansky, Nanoscale 2014, 6, 1580.

[49] K. Chong, S. D. Kelly, S. Smith, J. D. Eldredge, Phys. Fluids 1994-Present 2013, 25, 33602.

[50] H. Faxén, Ark Mat Astron Fys 1924, 18, 1.

[51] P. G. Saffman, J. Fluid Mech. 1965, 22, 385.

[52] M. R. Maxey, J. J. Riley, Phys. Fluids 1958-1988 1983, 26, 883.

[53] J. J. Paszkowiak, A. Dardik, Vasc. Endovascular Surg. 2003, 37, 47. 


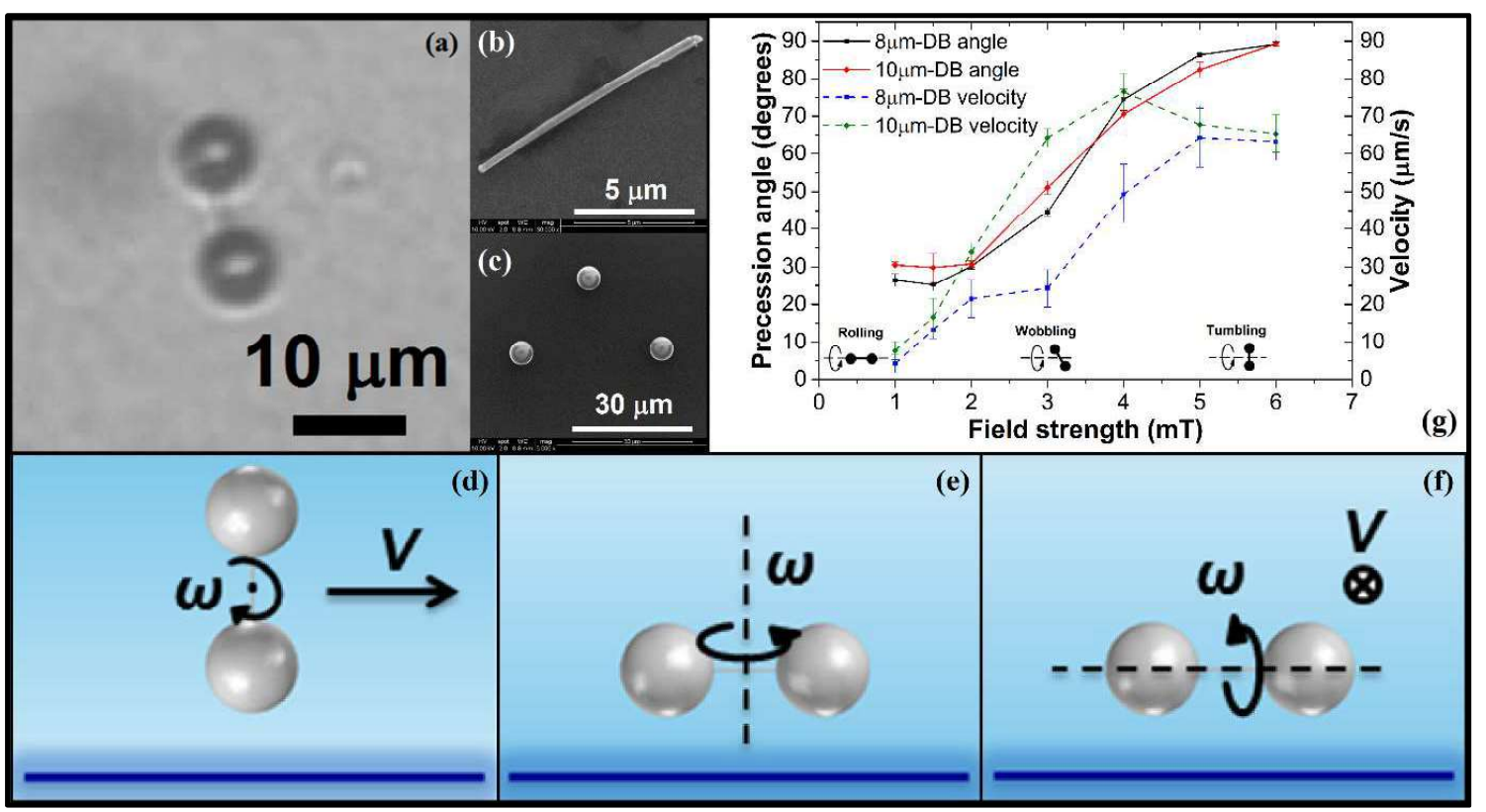

Figure 1. (a) Optical image of a magnetic DB assembled by a Ni NW and two PS microbeads (see Supporting Information Video V1 for the assembly process). The body length of the DB is defined as the center-to-center distance of its two microbeads. Due to the non-uniform length of NWs as well as randomness of the current assembly process, the size of individual DBs may vary. (b) (c) are SEM (scanning electron microscope) images of one Ni NW and the PS microbeads that have been used, the scale bars on the bottom right of which represent $5 \mu \mathrm{m}$ and $30 \mu \mathrm{m}$, respectively. (d) Translational control: DB tumbling with net propulsion in a vertical plane perpendicular to the wall. (e) Static control: DB spinning locally in a horizontal plane parallel to the wall. (f) Rolling motion of the DB. The direction of the translation velocity is pointing into the plane of the paper. $(\mathrm{g})$ Dynamics of an $8 \mu \mathrm{m} \mathrm{DB}$ and a $10 \mu \mathrm{m}$ DB in a rotating magnetic field of $20 \mathrm{~Hz}$. The precession angles and velocity values are measured (via Image $\mathrm{J}($ ) and calculated from the extracted frames of experiment videos (for the $8 \mu \mathrm{m} \mathrm{DB}$, see Supporting Information Video V2). Error bars for angles represent stand deviation from three measurements, and error bars for velocities represent the measurement difference of counting 5 more or less image pixels. 


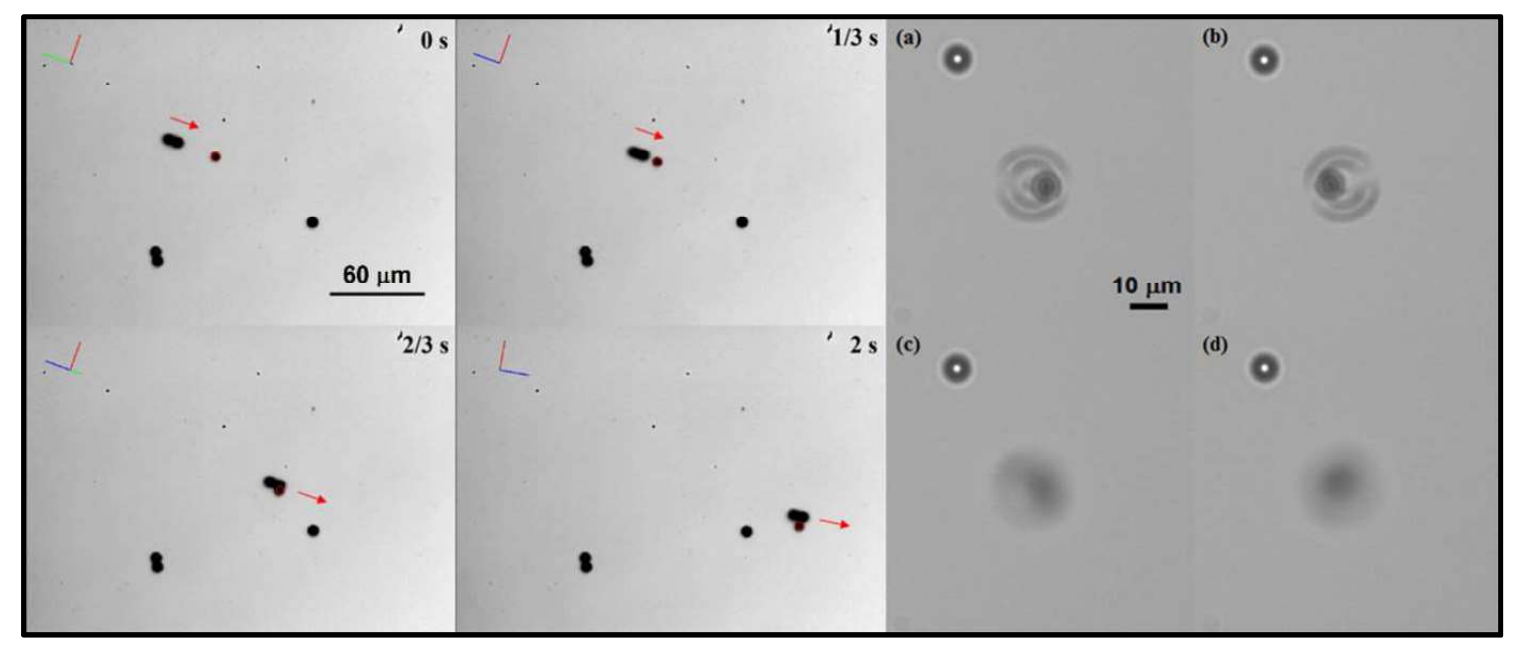

Figure 2. Left panel (0-2 s): trapping and transport microbead with tumbling DB FT under a magnetic field of $5 \mathrm{mT}, 20 \mathrm{~Hz}$ (see Supporting Information Video V4). From $0 \mathrm{~s}$ to $1 / 3 \mathrm{~s}$, the DB tumbles tangentially to the microbead; from $1 \mathrm{~s}$ to $2 \mathrm{~s}$, the microbead is trapped and translates with the DB. The red axis on the upper left indicates the rotation axis of the magnetic field. The microbead for trapping is outlined with a red circle, and the advancing direction of the DB is indicated by a red arrow. Right panel (a-d): microbead originally orbiting the microvortex above the rotating DB at moderate frequencies migrates inward as the rotation frequency of the magnetic field is abruptly increased to frequencies above $20 \mathrm{~Hz}$ (with the field strength fixed at $5 \mathrm{mT}$ ). The bright spot on the upper left is an identical PS microbead ( $6 \mu \mathrm{m}$ in diameter) to the one trapped in microvortex, which remains undisturbed by the rotational flows induced nearby the DB. The blurring effect in (c) (d) is presumably caused by the lift of the DB in the vertical direction due to rotation at frequencies much higher than $20 \mathrm{~Hz}$. 


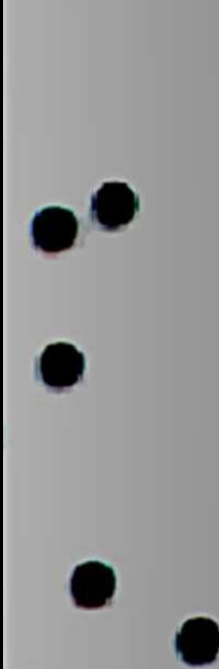

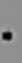
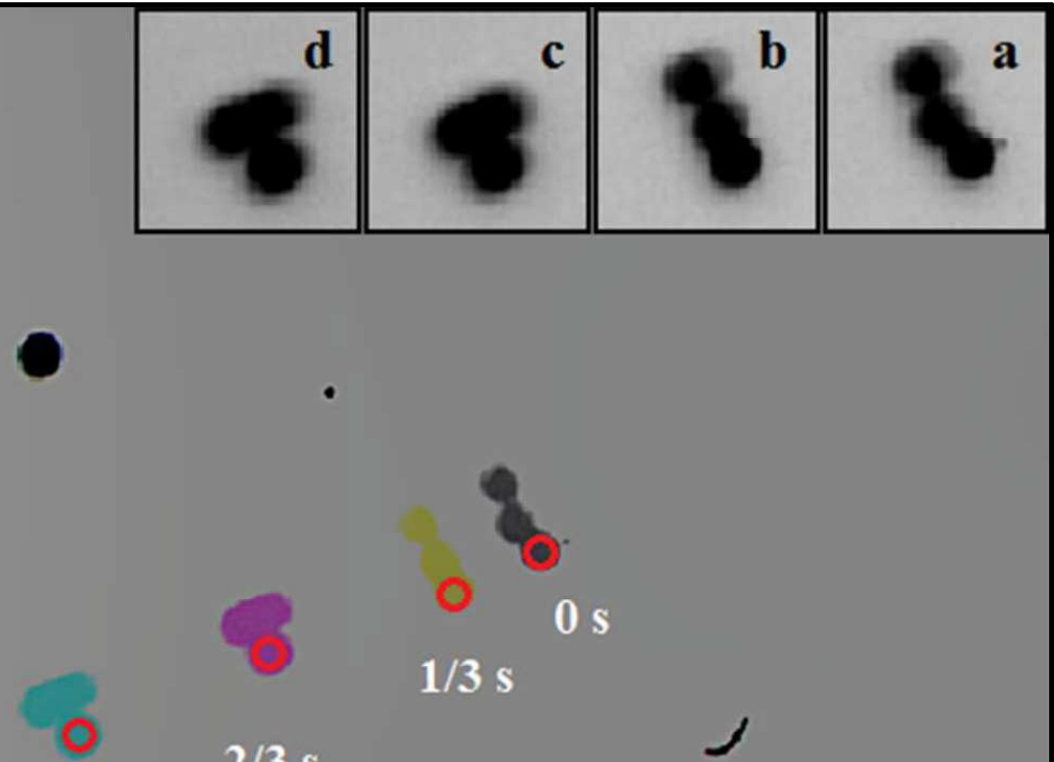

\section{$1 \mathrm{~s}$}
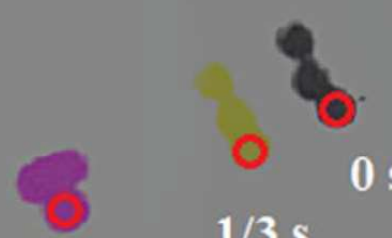

$0 \mathrm{~s}$

\section{$1 / 3 \mathrm{~s}$}

\section{$2 / 3 \mathrm{~s}$}

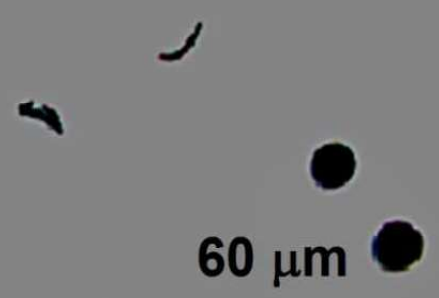

Figure 3. Superimposed images (via Image ${ }^{\circledR}$ ) showing the transition of DB FT from rolling trapping to tumbling trapping under a rotating magnetic field of $30 \mathrm{~Hz}$ (see Supporting Information Video V5). The field strength for $0 \mathrm{~s}, 1 / 3 \mathrm{~s}, 2 / 3 \mathrm{~s}$ and $1 \mathrm{~s}$ is $2.5 \mathrm{mT}, 3 \mathrm{mT}, 7.5 \mathrm{mT}$ and $12 \mathrm{mT}$, respectively. The DB and trapped microbead at different time points are marked with varying colors. Also, the microbead is outlined with a red circle. The insets $a, b, c, d$ show the enlarged view of the DB with the trapped microbead at $0 \mathrm{~s}, 1 / 3 \mathrm{~s}, 2 / 3$ and $1 \mathrm{~s}$, respectively. 


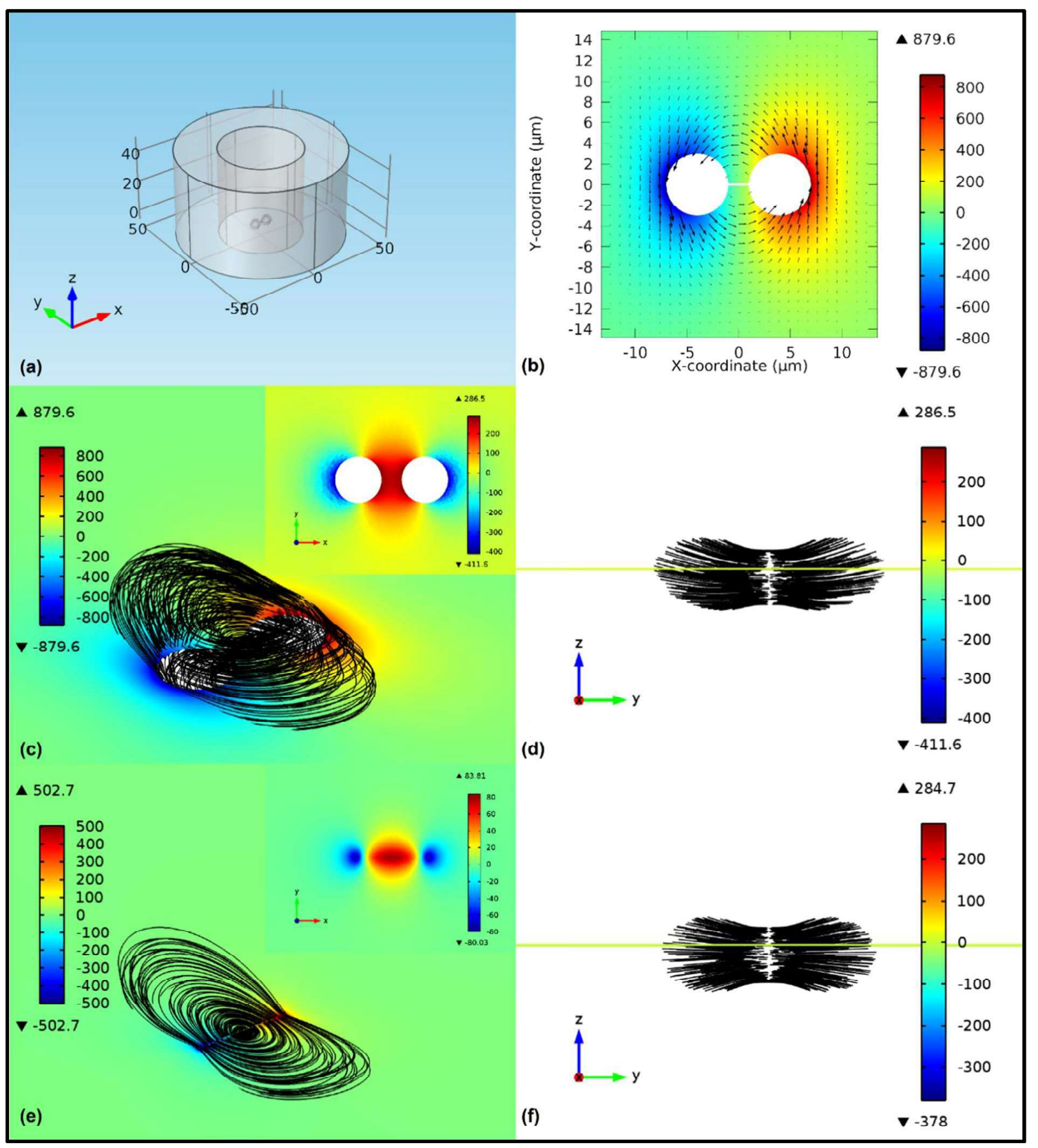

Figure 4. (a) Geometry of the FEM model for a rotating DB above a no-slip wall. The rotation axis is defined as Z-axis, and the rotation plane the XY-plane. The numbers represent dimensions in micrometer. (b) Tangential flow induced by the DB rotating at a frequency of $20 \mathrm{~Hz}$ in a plane $6 \mu \mathrm{m}$ above the wall. The horizontal/vertical axes represent spatial coordinates $(\mu \mathrm{m})$. The contour shows magnitude of the tangential velocity $(\mu \mathrm{m} / \mathrm{s})$, marked by the color legend on its right hand side. And the arrows in it indicate the flow field in the rotation plane. (c)-(f): The deformation of streamlines for an $8 \mu \mathrm{m}$-long DB or NW rotating at $20 \mathrm{~Hz}$. (c) (d) (e) is with the presence of a no-slip wall $6 \mu \mathrm{m}$ below the rotating body, and (f) is in unbound fluid. The color legends in the main frame of (c) (e) illustrate the magnitude of tangential velocities $(\mu \mathrm{m} / \mathrm{s})$ in the rotation plane, and those in (d) (f) and the insets of (c) (e) illustrate the magnitude of z-component vorticity in a plane $1 \mu \mathrm{m}$ above the rotation plane, marked by a straight yellow line in (d) (f). 


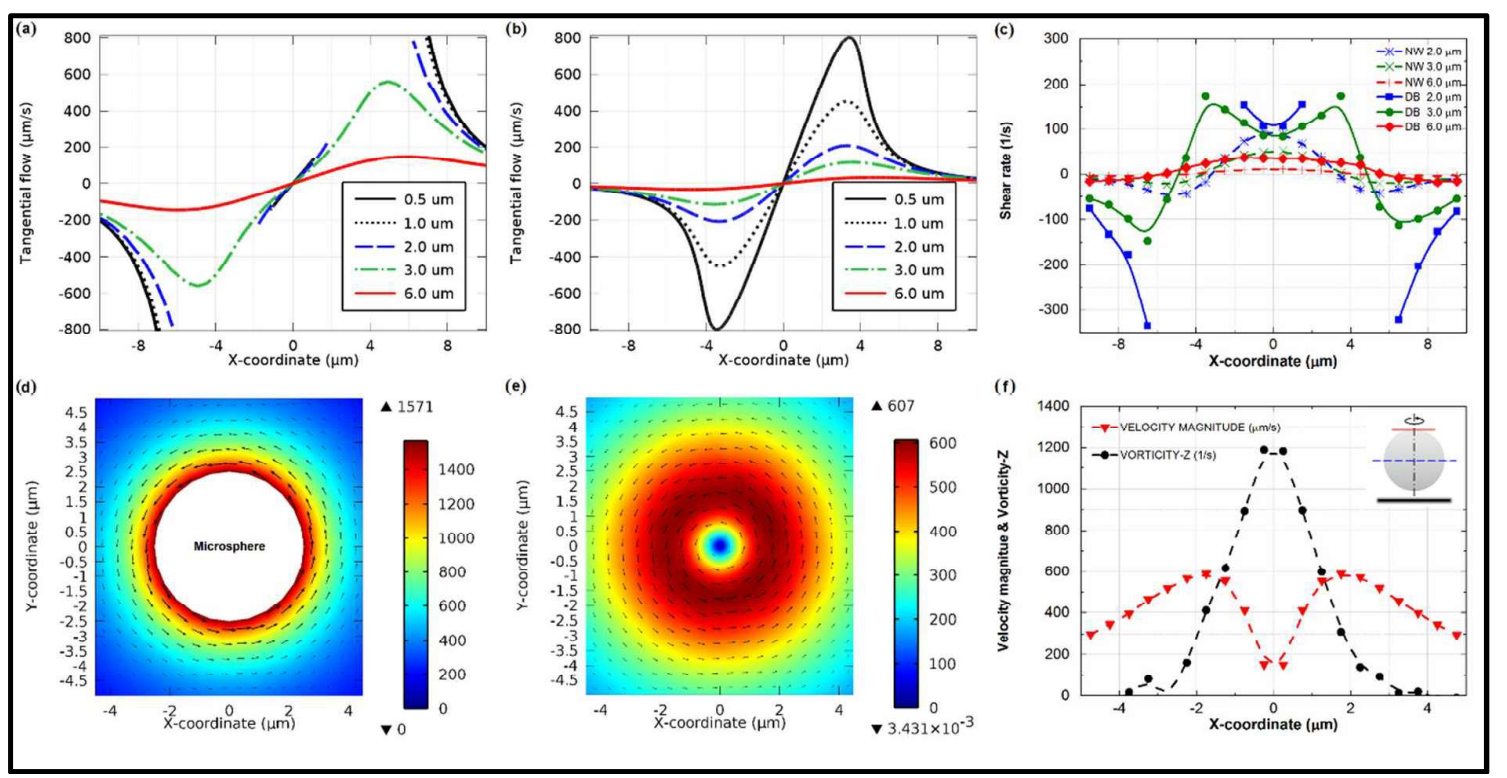

Figure 5. (a)-(c): Tangential flows and the associated shear rates in parallel planes $(Z=0.5$ $\mu \mathrm{m}, 1.0 \mu \mathrm{m}, 2.0 \mu \mathrm{m}, 3.0 \mu \mathrm{m}, 6.0 \mu \mathrm{m})$ above the rotation plane $(\mathrm{Z}=0 \mu \mathrm{m})$ of a $8 \mu \mathrm{m} \mathrm{DB}$ rotating at $20 \mathrm{~Hz}$ and a $8 \mu \mathrm{m} \mathrm{NW}$ rotating at $95 \mathrm{~Hz}$, respectively. The profiles in (a) and (b) show y-component velocities (i.e., the tangential velocities) at different positions along the Xaxis direction, and have been produced in COMSOL Multiphysics ${ }^{\circledR}$. Missing segments of profiles at $\mathrm{Z}=0.5 \mu \mathrm{m}, 1.0 \mu \mathrm{m}$ and $2.0 \mu \mathrm{m}$ in (a) is due to the existence of two spheres (radius: $3 \mu \mathrm{m}$ ), whose centers are positioned at $X=-4 \mu \mathrm{m}, 4 \mu \mathrm{m}$. The profiles in (c) selectively show shear rates $\left(\mathrm{s}^{-1}\right)$ in parallel planes $(\mathrm{Z}=2.0 \mu \mathrm{m}, 3.0 \mu \mathrm{m}, 6.0 \mu \mathrm{m})$ above the rotation plane. The profiles in this plot are produced using Origin ${ }^{\circledR}$ based on the data points extracted from the simulation results of COMSOL Multiphysics ${ }^{\circledR}$. Again, missing segments of the profile at $Z=$ $2.0 \mu \mathrm{m}$ for DB is due to the existence of two spheres (radius: $3 \mu \mathrm{m}$ ), whose centers are positioned at $\mathrm{X}=-4 \mu \mathrm{m}, 4 \mu \mathrm{m}$. (d)-(f): Rotational flows induced by a $5 \mu \mathrm{m}$ sphere spinning at $100 \mathrm{~Hz}$. (d) The equatorial plane, which corresponds to the blue dash line in the inset of (f). (e) A tangent plane parallel to the equatorial plane, which correspond to the red solid line in the inset of (f). The color legend next to both contours shows the velocity magnitude $(\mu \mathrm{m} / \mathrm{s})$ in the plane, and the arrows indicate velocity directions. (f) Distributions of velocity magnitude and z-component vorticity in the plane of (e), the profiles in which are produced using

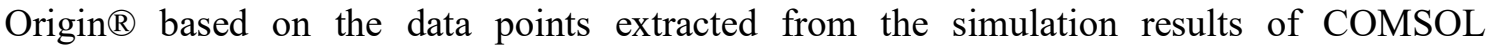
Multiphysics ${ }^{\circledR}$. 

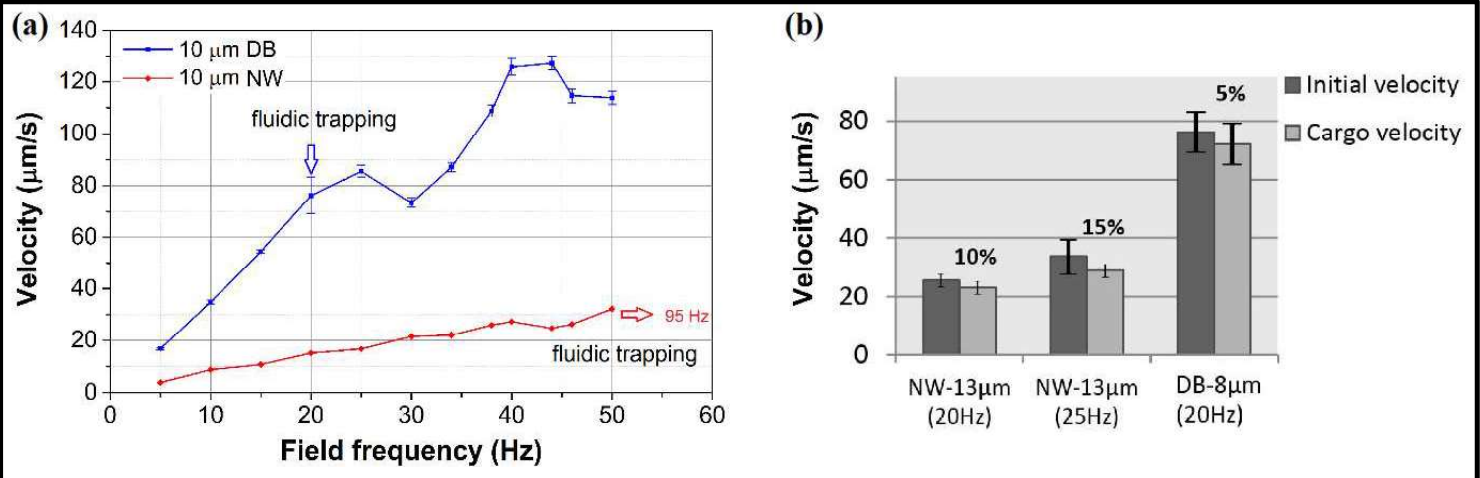

Figure 6. (a) Frequency-velocity relationship for a $10 \mu \mathrm{m}$ magnetic DB and $10 \mu \mathrm{m}$ Ni NW under a magnetic field of $5 \mathrm{mT}$. Data for the NW are extracted from Zhang et al.. ${ }^{[7]}$ The arrows indicate the frequency observed in experiment above which fluidic trapping occurs. Error bars within $5-20 \mathrm{~Hz}$ (corresponding to small and medium translation velocities) count for the measurement difference of 5 image pixels, and those within $20-50 \mathrm{~Hz}$ (corresponding to relatively large translation velocities) count for the measurement difference of 10 image pixels. (b) Velocity loss due to cargo loading of a $6 \mu \mathrm{m}$ PS microbead using NW FT and DB FT in tumbling trapping mode. The magnetic field strength above is 3 $\mathrm{mT}$ for NWs and $5 \mathrm{mT}$ for the $\mathrm{DB}$, respectively, under which both microactuators assume tumbling motion and can be reasonably compared. Note that the length $8 \mu \mathrm{m}$ of DB refers to its center-center distance. Its total length should be about $14 \mu \mathrm{m}$, which is comparable to the listed NWs'. Data of the NWs for comparison are extracted from Petit et al.. ${ }^{[42]}$ 


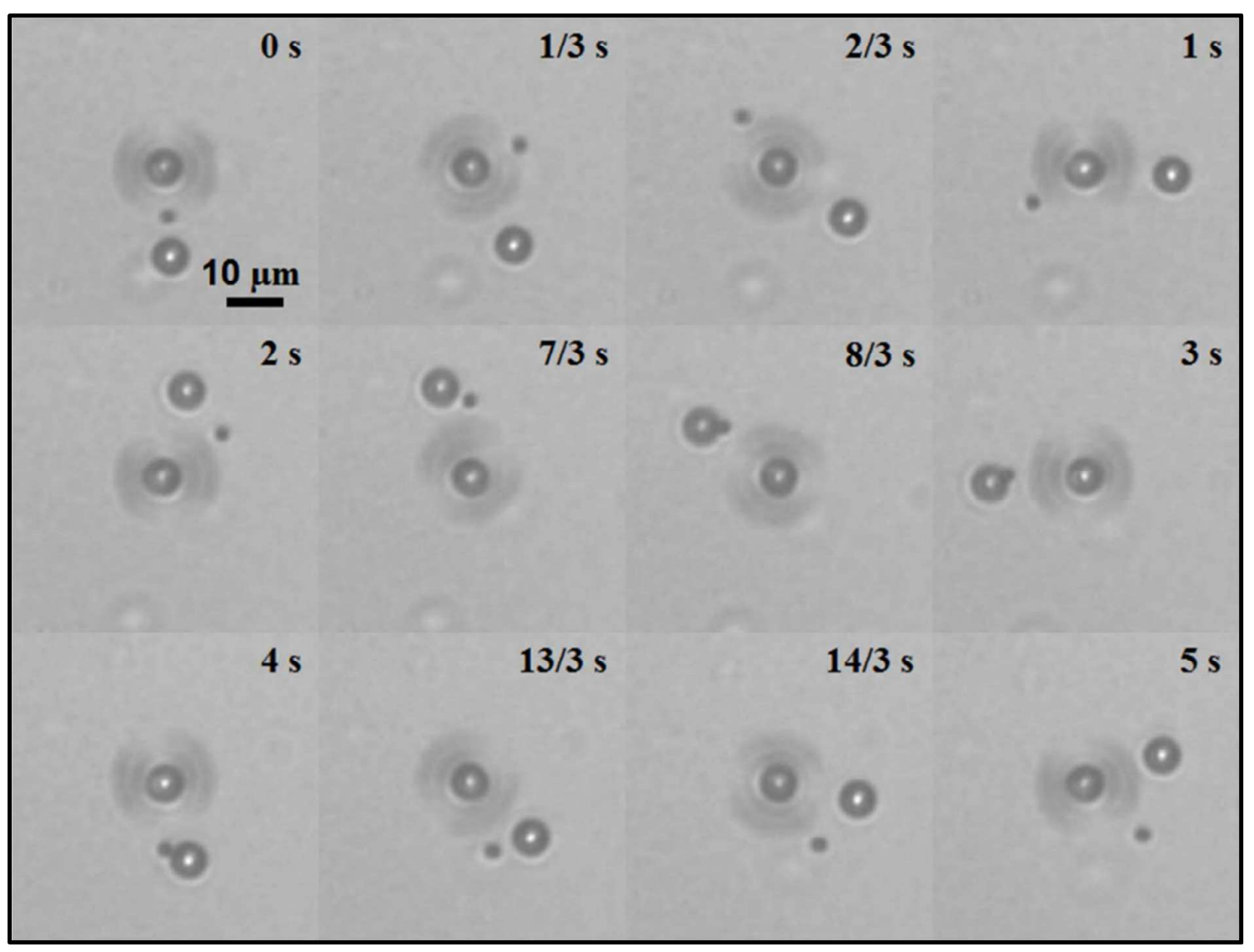

Figure 7. PS microbead and yeast cell trapped in the rotational flows of the microvortex above a rotating DB. Inside the microvortex is another PS microbead that has been immobilized by the fluidic trap. From 0 to $1 \mathrm{~s}$, the yeast cell is chasing the microbead in an orbit of smaller diameter; from $2 \mathrm{~s}$ to $3 \mathrm{~s}$, the yeast cell is affected by the secondary flow nearby the microbead and consequently trapped by the it; from $4 \mathrm{~s}$ to $5 \mathrm{~s}$, the yeast is detached from the microbead and moves in the same orbit with it. 


\begin{tabular}{ccccc}
\hline Time & $0 \mathrm{~s}$ & $1 / 3 \mathrm{~s}$ & $2 / 3 \mathrm{~s}$ & $1 \mathrm{~s}$ \\
\hline position-M (deg) & $-85.07 \pm 5$ & $-60.75 \pm 5$ & $-35.66 \pm 5$ & $-3.01 \pm 5$ \\
position-Y (deg) & $-84.64 \pm 5$ & $23.63 \pm 5$ & $126.87 \pm 5$ & $213.69 \pm 5$ \\
speed-M (rad/s) & & $1.27 \pm 0.09$ & $1.31 \pm 0.09$ & $1.71 \pm 0.09$ \\
speed-Y (rad/s) & & $5.67 \pm 0.09$ & $5.41 \pm 0.09$ & $4.55 \pm 0.09$ \\
\hline Time & $2 \mathrm{~s}$ & $7 / 3 \mathrm{~s}$ & $8 / 3 \mathrm{~s}$ & $3 \mathrm{~s}$ \\
\hline position-M (deg) & $73.78 \pm 5$ & $108.73 \pm 5$ & $146.58 \pm 5$ & $185.53 \pm 5$ \\
position-Y (deg) & $394.02 \pm 5$ & $450.00 \pm 5$ & $497.56 \pm 5$ & $538.83 \pm 5$ \\
speed-M (rad/s) & & $1.83 \pm 0.09$ & $1.98 \pm 0.09$ & $2.04 \pm 0.09$ \\
speed-Y (rad/s) & & $2.93 \pm 0.09$ & $2.49 \pm 0.09$ & $2.16 \pm 0.09$ \\
\hline Time & $4 \mathrm{~s}$ & $13 / 3 \mathrm{~s}$ & $14 / 3 \mathrm{~s}$ & $5 \mathrm{~s}$ \\
\hline position-M (deg) & $287.74 \pm 5$ & $318.01 \pm 5$ & $348.91 \pm 5$ & $379.44 \pm 5$ \\
position-Y (deg) & $630.00 \pm 5$ & $649.57 \pm 5$ & $663.02 \pm 5$ & $677.42 \pm 5$ \\
speed-M (rad/s) & & $1.58 \pm 0.09$ & $1.62 \pm 0.09$ & $1.60 \pm 0.09$ \\
\hline speed-Y (rad/s) & & & $0.70 \pm 0.09$ & $0.75 \pm 0.09$ \\
\hline & & & & \\
\hline
\end{tabular}

Table 1. Azimuthal position and angular speed of the PS microbead and yeast cell as shown in Figure 7. The positions of both the microbead and yeast are indicated by timevarying angles, which are measured in an accumulative manner to facilitate the calculation of instantaneous angular speed. The instantaneous angular speed is calculated as the change of radian per $1 / 3$ seconds. Based on previous errors of angle measurement (refer to Figure 1g), the standard deviation is set as \pm 5 for azimuthal position, resulting in a deviation of $\pm \mathbf{0 . 0 9}$ for the instantaneous angular speed. For brevity, the microbead is referred to as " $M$ " and yeast as " $Y$ " in this table. 


\section{Graphical table of contents (ToC)}

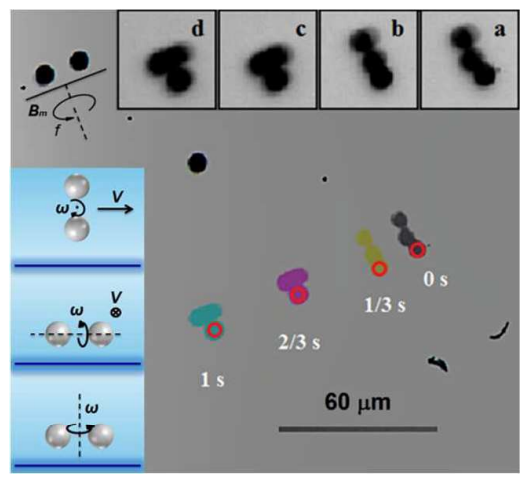

ToC entry: A dumbbell microactuator consisting of nickel nanowire and polystyrene microbeads is developed as a type of versatile fluidic tweezers to trap and transport individual microobjects. It can generate robust and highly focused microvortices when actuated with a low-strength rotating magnetic field, and is able to manipulate microbojects with high precision via three-modal locomotion, namely rolling, wobbling, and tumbling. 